\title{
Kernos
}

Revue internationale et pluridisciplinaire de religion grecque antique

$28 \mid 2015$

Varia

\section{Le Sacrifice humain dans tous ses états}

\section{Stella Georgoudi}

\section{(2) OpenEdition \\ Journals}

Édition électronique

URL : https://journals.openedition.org/kernos/2339

DOI : 10.4000/kernos.2339

ISSN : 2034-7871

\section{Éditeur}

Centre international d'étude de la religion grecque antique

\section{Édition imprimée}

Date de publication : 1 octobre 2015

Pagination : 255-273

ISBN : 978-2-87562-055-2

ISSN : 0776-3824

\section{Référence électronique}

Stella Georgoudi, «Le Sacrifice humain dans tous ses états », Kernos [En ligne], 28 | 2015, mis en ligne le 01 octobre 2015, consulté le 21 septembre 2021. URL : http://journals.openedition.org/kernos/2339 ; DOI : https://doi.org/10.4000/kernos.2339

Ce document a été généré automatiquement le 21 septembre 2021.

Kernos 


\title{
Le Sacrifice humain dans tous ses états
}

\author{
Stella Georgoudi
}

\section{RÉFÉRENCE}

Pierre BonNeCHERE, Renaud GAGNÉ (éd.), Sacrifices humains. Perspectives croisées et représentations / Human sacrifice. Cross-cultural perspectives and representations, Liège, Presses Universitaires de Liège, 2013. 1 vol. $16 \times 24$ cm, 266 p., 16 pl. (Religions. Comparatisme - Histoire - Anthropologie, 2). ISBN : 978-2-87562-021-7

Agnès A. NAGY, Francesca PRESCENDI, Sacrifices humains. Dossiers, discours, comparaisons, Actes du colloque de Genève, 19-20 mai 2011, Turnhout, Brepols, 2013. 1 vol. $15,5 \times 23,5$ cm, 280 p. (Bibliothèque de l'École des Hautes Études, Sciences religieuses, 160). ISBN : 978-2-503-54802-8.

1 Trois ouvrages - deux collectifs ${ }^{1}$ et une monographie ${ }^{2}-$, publiés ces dernières années, méritent une attention toute particulière. Ces trois livres, riches, variés, stimulants, sont, en effet, consacrés à deux thèmes aussi importants qu'embarrassants en histoire des religions : le sacrifice humain et l'anthropophagie. Deux sujets qui ne cessent d'intriguer, de fasciner, d'horrifier même certains esprits d'hier et d'aujourd'hui, en les faisant parfois osciller entre l'acceptation naturelle (argumentée ou pas) et la négation en bloc de ces actes dont témoignent plusieurs cultures - qu'ils soient des actes réels, ou imaginaires, ou symboliques.

2 Le sacrifice humain est surtout à l'honneur dans les deux ouvrages collectifs, structurés chacun selon ses propres choix et objectifs. Les éditeurs et éditrices de ces mises en scène communes n'abordent pas ce sujet en "novices" - loin de là ! Pierre Bonnechere a depuis longtemps aiguisé les couteaux sacrificiels, en se lançant vaillamment dans les routes tortueuses du sacrifice humain ${ }^{3}$. Dans son exploration minutieuse du sacrifice chez les Romains, Francesca Prescendi a prêté une oreille attentive au discours des érudits romains sur l'immolation des êtres humains ${ }^{4}$. Agnès Nagy a eu le courage de 
regarder en face un des "monstres", comme elle dit, les plus redoutables: l'anthropophage et le cannibale, ce qui l'a amenée à poser souvent la question du sacrifice humain, comme nous allons le voir. Quant à Renaud Gagné, son dernier livre (Ancestral Fault in Ancient Greece, Cambridge, 2013) ne manquera pas de nourrir la réflexion de qui s'intéresse à la relation entre sacrifice humain et fautes des mortels (qu'elles soient ancestrales ou non).

\section{Un cannibalisme raconté à travers les âges}

Essayons donc de parler de ces trois ouvrages - pour autant qu'une présentation de dossiers aussi étoffés et pluriculturels soit possible - en commençant par la monographie d'A. Nagy. Divisé en quatre grandes parties (1. «La vie sous Cronos»: le mythe des races et le sacrifice; 2 . Le règne de Dionysos ; 3 . L'Autre; 4 . Juifs et chrétiens face aux rumeurs), cet ouvrage pose d'emblée les bonnes questions: Pourquoi l'anthropophagie, le fait de manger de la chair humaine " éveille-t-elle tant d'émotion, tant de haine?». Pourquoi le cannibalisme, l'acte de consommer «les membres de sa propre espèce ", soulève-t-il "cette terreur mêlée de fascination», pourquoi provoque-t-il «le sentiment de danger que l'Autre suscite en nous-mêmes?». Pour essayer de trouver quelques réponses à ces interrogations, A. Nagy s'engage dans un long et difficile chemin qui l'amène, de l'époque de Kronos et du mythe hésiodique des races, au judaïsme et au christianisme. Un chemin escarpé où elle rencontre l'orphisme et le dionysisme, des récits sur « les mères meurtrières » (les filles de Cadmos, de Proïtos ou de Minyas), mais aussi sur « les pères meurtriers » (tels Lycurgue, Athamas ou Lycaon), avant d'affronter "l'Autre", sous les traits non seulement du "barbare", mais également du «tyran ", du " conjuré ", du "révolté », ou encore "des groupements religieux » (magiciens, devins ou philosophes, tels les cyniques et les stoïciens), qui se placent « hors des cadres civiques ».

Un tel parcours, qui dure plus de mille ans et qui traverse la Grèce des cités, les royaumes hellénistiques, l'Empire romain, voire surtout l'Empire romain chrétien, sans oublier, sur la route, Israël, un tel trajet donc requiert un effort sans relâche, une connaissance des sources approfondie, et A. Nagy n'a pas manqué à cette lourde tâche. Mais à cause sans doute de sa formation dans la Faculté de Théologie et de Sciences des Religions (Université de Lausanne), elle est manifestement plus à l'aise lorsqu'elle explore l'anthropophagie et le cannibalisme dans l'espace du judaïsme et de la chrétienté de l'Empire romain. Dans ce domaine, elle fait preuve d'une connaissance aiguisée et directe des textes et de la bibliographie, ses analyses sont plus pertinentes, ses réflexions plus subtiles ${ }^{5}$.

De ce long voyage fructueux, essayons de résumer les points essentiels présentés, en espérant ne pas trahir la pensée de l'auteure. Tout d'abord, à travers des récits, des mythes, des critiques ou des réfutations, le cannibalisme est souvent associé à l'immolation d'êtres humains, à commencer par l'histoire de Kronos et de son équivalent romain Saturne, figures emblématiques, en Grèce et en Italie, des fêtes Cronia et Saturnalia, auxquelles "bon nombre de sacrifices humains sont liés». Ensuite, l'analyse de quatre mythes, avec comme protagonistes Lycaon et Tantale, Thyeste et Térée, montre les difficultés exprimées par le cannibalisme pour « la mise en place des règles de la vie civilisée ». Mais pour ces « quatre héros » le temps n'est pas le même. Lycaon et Tantale «appartiennent à la génération où les frontières entre 
hommes et dieux sont en train de se dessiner ». Par le « repas cannibale», ils veulent " sonder » les pouvoirs des dieux dont ils « mettent en doute les prérogatives ». Thyeste et Térée « vivent dans un monde où les règles sont déjà en place, mais ils ne respectent pas ». Quoi qu'il en soit, le « cannibalisme » de ces quatre figures est « le symbole de la transgression définitive » qui exclut absolument le « retour à la normalité ».

6 Avec la figure de Dionysos, les problèmes d'interprétation deviennent beaucoup plus importants. Mais pour A. Nagy, la présence de ce dieu, inséparable du mythe de «son démembrement", est partout associée à "des récits cannibales» (sur Penthée ou Lycurgue, sur Proétides ou Minyades). En abordant les questions que posent l'orphisme et le dionysisme, l'auteure discute et nuance la thèse de Marcel Detienne, pour qui l'orphisme et le pythagorisme seraient « diamétralement opposés » au dionysisme et au cynisme, bien qu'il s'agisse de "quatre mouvements contestataires de l'ordre politicoreligieux de la cité ». Pour A. Nagy, « orphisme et dionysisme ne sont pas des courants contradictoires, s'excluant mutuellement ", et on ne saurait réduire ces mouvements à une opposition entre les partisans d'un végétarisme pur et ceux qui pratiqueraient « uniquement des rites ômophagiques ». Reste à situer dans le temps le «cannibalisme dionysiaque ", à travers l'histoire de la "cuisine titanique » et des châtiments infligés par Dionysos à ses opposants, en les rendant cannibales, mangeurs de leurs propres enfants. Or, ces châtiments n'appartiennent pas à un passé révolu, ils interviennent chaque fois qu'on refuse "d'honorer le dieu de la mania", ce qui signifie que l'anthropophagie qui «surgit à la suite» du dieu «n'est pas une réminiscence du monde pré-civilisé ». Et A. Nagy de conclure : « l'ère de Dionysos ne précède pas celle de la cité, elle lui est parallèle ».

7 Considérée par les Grecs et les Romains comme «un crime et une souillure inexpiables ", l'anthropophagie est souvent attribuée à l'Autre, par rapport auquel les peuples «civilisés » doivent se définir. Et cet «Autre » peut appartenir à un passé mythique, comme les Cyclopes, avec leurs "goûts cannibales", ou à un présent "observable", comme certains peuples barbares, tels les Massagètes, les Padéens en Inde, et surtout les Androphagoi, ces "Mangeurs d'hommes », qui ne reconnaissent dit Hérodote - aucune justice et n'utilisent aucune loi. Certes tous les barbares ne sont pas cannibales ou, au moins, pas «de vrais cannibales», comme pourrait le montrer le cas des Scythes, qui ne pratiquent l'anthropophagie "qu'occasionnellement». Cependant, cet « Autre » peut aussi prendre les traits d'un tyran, d'un monarque, qu'il subisse un "cannibalisme forcé » (comme Kyaxare), ou qu'il fasse manger à quelqu'un de la chair humaine (comme Astyage). Enfin, les conjurés, les révoltés et d'autres groupes subversifs et anti-sociaux (ou considérés comme tels: magiciens, sorciers, devins itinérants etc.) n'échappent pas au soupçon du cannibalisme, tandis que certains philosophes ou écoles philosophiques (avant tout les cyniques et les stoïciens) «sont suspectés de défendre le cannibalisme ", non pas à cause de leurs " pratiques ", mais en raison de leurs « enseignements".

8 Imaginé presque toujours comme "un repas sacrificiel», le cannibalisme est relégué ainsi dans un passé lointain et révolu des peuples «civilisés", mais surtout dans un ailleurs «barbare", chez des méchants et des impies, chez nos ennemis et adversaires qui ne nous ressemblent pas. Il constitue donc une arme accusatrice redoutable pour attaquer les « Autres ", pour les dénigrer, pour les rejeter hors de l'humanité, pour les exclure définitivement. On voit ainsi des Romains s'élever contre des Égyptiens " cannibales ", ou contre des Juifs, chefs de la révolte, qui boivent «le sang de leurs 
concitoyens " et se partagent leurs cadavres; on assiste aux "trois accusations " virulentes $(\dot{\varepsilon} \gamma \kappa \lambda \eta ́ n \alpha \tau \alpha)$, portées par les païens contre les chrétiens, trois « crimes » : à savoir, «l'athéisme, les repas de Thyeste (l'anthropophagie), les unions œedipiennes (l'inceste) »; et finalement, avec l'avènement et la victoire du christianisme, par une sorte de détournement de la situation et de ses acteurs, ce sont les auteurs chrétiens et l'Église naissante qui vont contre-attaquer, en brandissant les mêmes armes à l'encontre non seulement des païens mais aussi des hérétiques. Les apologistes, de langue grecque ou latine, "assimilant les anciens dieux aux mauvais démons", mélangeant des récits grecs, romains et barbares portant sur des meurtres ou le cannibalisme rituel, qualifient de "corrompue » la civilisation hellénique, considèrent que la paideia grecque est "tournée vers l'anthropophagie», bref, ils croient fermement que «seul le christianisme est capable d'empêcher l'humanité de sombrer dans le chaos symbolisé par le sacrifice humain et le cannibalisme ».

Le grand nombre de thèmes abordés dans cet ouvrage, la longue période parcourue, les confrontations explorées entre païens, Juifs et chrétiens, rendent impossible ici la discussion approfondie qu'aurait mérité ce travail. Je dirais seulement, pour clore cette brève présentation, qu'il n'est pas toujours aisé de suivre l'auteure, lorsqu'elle navigue entre textes grecs et latins, quand, à propos, par exemple, de mythes, de personnages, de faits rituels, elle présente sans trop contextualiser des versions séparées dans l'espace et le temps, et de façon souvent significative. Par ailleurs - et je m'arrête ici certaines théories, opinions, affirmations exprimées et formulées, au fil des années, par certains spécialistes sont adoptées sans discussion ultérieure par l'A., bien qu'elles aient été, entretemps, mises en question par d'autres (l'idée, par exemple, que « seuls les hommes ont le droit d'accomplir une thysia » [p. 84], ou encore l'image stéréotypée d'un Dionysos «toujours ressenti comme un nouveau venu dans la cité, un Grec qui a passé sa vie en Orient, parmi les Barbares » [p. 103, c'est moi qui souligne], comme si ce dieu à plusieurs facettes et fonctions, à la manière d'ailleurs de toutes les divinités du polythéisme, était façonné exclusivement d'après la figure choisie et imposée par les Bacchantes d'Euripide).

\section{Le sacrifice humain : d'une culture à l'autre}

L'ouvrage collectif, édité et introduit par Pierre Bonnechere et Renaud Gagné, comprend 10 contributions qui donnent la priorité aux Grecs, tout en mettant en regard deux autres cultures fort éloignées, la Chine et les Aztèques. En fin de parcours, on revient de nouveau à la Méditerranée antique, pour céder la parole aux Romains à travers une grande épopée, l'Énéide de Virgile. On a voulu, dans ce volume, mettre l'accent non pas sur l'historicité du sacrifice humain, ou sur la recherche de quelques "survivances" du passé, mais sur « le thème de la représentation du sacrifice humain ", considéré comme un sujet plus intéressant et prometteur. Cette option de la représentation, qui permettait, selon les éditeurs, «d'échapper à la difficulté de la définition du phénomène dans l'absolu» (p.13), facilitait aussi la comparaison entre aires culturelles différentes, en se fondant ainsi «sur des témoignages du même ordre », en se situant dans le domaine des perceptions et des représentations.

11 Dans son texte "Victime humaine et absolue perfection dans la mentalité grecque ", qui ouvre la série des contributions, Pierre Bonnechere revient, en guise d'introduction, sur une thèse qu'il a déjà développée et défendue ailleurs : d'Homère 
jusqu'à « la fin de la littérature grecque, au ve siècle apr. J.-C. », le sacrifice humain serait considéré et présenté comme "contraire aux règles des Grecs", comme une "anti-norme", pourrait-on dire, un acte enfin propre aux barbares. Dans ce texte cependant, il entend dresser avec minutie l'image de la victime humaine, comme elle ressort des sources grecques écrites, dont la tragédie occupe une bonne place. Au même titre que l'animal sacrificiel doit être sans défaut, «pur » (katharon), beau (voire même kalliston), on exige souvent que la victime humaine soit belle, jeune, mais aussi « de bonne naissance ", de "sang noble ». À partir de la seconde moitié du v viècle, remarque P. Bonnechere, c'est également la «noblesse morale » qui compte, lorsqu'on voit apparaître le thème du " courage patriotique d'affronter la mort ", pour sauver la patrie. Mais l'auteur jette aussi un éclairage particulier sur «l'idée d'offrir des enfants innocents", en observant qu'il est "assez rare» que la victime humaine "soit responsable de l'impiété qui cause le courroux divin ». Cependant, bien que la victime humaine soit figurée comme la victime animale, P. Bonnechere attire l'attention sur toute une série de subtilités, de nuances, voire de variations entre les deux types de victimes. Enfin, les changements dans les réalités politiques et idéologiques, intervenus au fil du temps, n'ont pas entamé la force d'une tradition qui voit dans le sacrifice de jeunes gens un moyen idéal pour représenter le passage à l'âge adulte et le mariage comme une mort symbolique et temporaire. - Cette contribution, pleine d'idées, mériterait une discussion détaillée, voire un débat contradictoire, qui n'a pas sa place dans cette notice ${ }^{6}$. Je signale seulement que la question essentielle, qu'on entrevoit souvent dans les études sur les "sacrifices humains", porte sur ce qu'on entend par cette expression, une question qui est posée plus clairement dans le livre collectif édité par Agnès Nagy et Francesca Prescendi (voir ci-dessous).

L'article de Ioannis Mylonopoulos (« Gory Details? The Iconography of Human Sacrifice in Greek Art ») apporte un éclairage particulier sur les représentations figurées de sacrifice humain. Il constate la rareté de ces images, le peu d'intérêt que montrent les peintres et sculpteurs grecs à ce thème, à travers les siècles, par rapport aux artistes étrusques et romains. Une rareté qui contraste étonnamment avec la profusion de textes littéraires, remplis d'histoires d'immolations d'êtres humains. Le sacrifice d'Iphigénie, par exemple, malgré la présence imposante d'un Euripide et de ses deux tragédies consacrées à la fille d'Agamemnon, ne trouve que peu d'écho dans l'art de la fin du $v^{e}$ siècle, comme aussi pendant le $\mathrm{IV}^{\mathrm{e}}$ siècle. En revanche, Polyxène, une figure beaucoup moins importante qu'Iphigénie dans la littérature, est plus souvent représentée sur des vases athéniens (surtout dans des scènes montrant le meurtre de Priam, ou l'embuscade de Troïlos). Deux scènes, cependant, figurant le sacrifice de Polyxène (la soi-disant amphore tyrrhénienne du peintre de Timiades et le " sarcophage de Polyxène »), sont particulièrement significatives à cause de la violence que subit la fille de Priam, portée et égorgée comme une bête, ainsi que le montre bien l'analyse de I. Mylonopoulos. Une remarque, cependant: le fait que Phoinix, un des personnages de l'amphore tyrrhénienne, se retourne et ne regarde pas l'acte sacrificiel ne signifie pas forcément la "rejection of the human sacrifice ", comme le pense l'auteur. En iconographie, il est risqué de "psychologiser" les regards, comme me le fait remarquer François Lissarrague, expert, comme on sait, en la matière. Enfin, ce que I. Mylonopoulos observe sur les images à partir de la fin du vi siècle, c'est une distinction qui se met en place entre, d'une part, les victimes humaines conduites à l'autel sans que leur mise à mort soit montrée, et, d'autre part, celles que l'auteur appelle « innocent victims » (tels Priam, Troïlos ou les enfants de Médée), dont «the 
sacrilegious killing... at the altar " représente «a semantically perverted version of human sacrifice... a clear distortion of the sacrificial order ». - Reste à savoir dans quelle mesure la mise à mort « sacrilège » des personnages cités peut être qualifiée de sacrifice humain, et encore ce que signifie exactement le "sacrifice perverti", une expression répétée souvent comme une évidence.

À travers sa contribution (« Human Sacrifice in Euripides' Iphigenia in Tauris: Greek and Barbarian »), Jan Bremmer nous plonge, avec subtilité, au cœur de trois sacrifices humains, en nous proposant comme guide Euripide et ses tragédies : trois sacrifices, dont les deux premiers mettent en scène la figure d'Iphigénie, celle d'Aulis et celle de Tauride, tandis que le troisième concerne son frère Oreste. Une des questions importantes que pose J. Bremmer est de savoir si Euripide dépeint le destin d'Oreste voué à être sacrifié chez les Taures, un peuple étranger donc - avec des couleurs différentes de celles d'Iphigénie dans la même pièce. En prêtant aussi attention au vocabulaire (thuô, sphazô, katakteinô, katarchomai, etc.), l'auteur nous fait suivre l'évolution de la pensée d'Euripide, d'une tragédie à l'autre, sa conception d'une divinité importante comme Artémis, la façon dont les barbares, en l'occurrence, les Taures, pratiquent le sacrifice humain, par rapport aux Grecs. J. Bremmer met en lumière les discordances, ou plutôt la polyphonie qui marque les passages examinés, et la façon dont Euripide organise les contradictions de certains personnages, en les combinant dans le discours tragique. Artémis, par exemple, règne sur un pays barbare, mais il n'y a rien de «strange or barbaric » dans cette déesse honorée par les Taures. Et si, aux dires d'Iphigénie, l'Artémis Taurique «prend du plaisir aux sacrifices

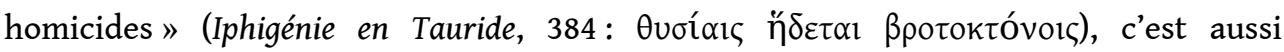
l'Artémis des Grecs, à Aulis, qui «se réjouit de victimes mortelles" (Iphigénie à Aulis,

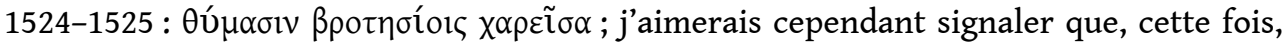
c'est le Chœur qui parle!) ${ }^{7}$. De l'autre côté, Thoas n'est pas seulement un «oriental despot », mais également «a pious Greek » qui obéit aux «paroles» (logous) d'Athéna, qui lui ordonne d'arrêter la poursuite des Grecs qui s'enfuient avec la «statue auguste » d'Artémis, « sa propre sœur ». Finalement, on pourrait dire qu'à lire Euripide à travers les analyses de J. Bremmer, les Taures ne sont pas ces "Barbares" odieux, écrasés par la "supériorité » de l'esprit grec, comme on l'a écrit ${ }^{8}$, selon une opinion binaire et récurrente qui oppose les "bons" Grecs aux "cruels" Barbares. Comme l'avait très bien dit Susanne Saïd, Euripide n'est " pas prisonnier du mythe de la supériorité grecque »"

C'est un texte particulièrement difficile à interpréter (voire même un texte corrompu, selon certains), qui se trouve au centre de la réflexion de Renaud Gagné («Athamas et Zeus Laphystios : Herodotus VII, 197 »). Il s'agit du seul passage des Histoires d'Hérodote où il est question d'un sacrifice humain, accompli en Grèce par des Grecs, à Alos (ou Halos), en Achaïe phthiotique, un acte associé au culte de Zeus Laphystios, maintes fois discuté, et que R. Gagné reconsidère attentivement dans le but de mettre en évidence " the logic of representation at work in the passage's extensive description of the sacrificial ritual» (p. 106). Les acteurs de ce rituel sont les descendants d'Athamas, sur lesquels pèse une terrible menace proférée par un "ordre divin » : si le presbutatos ${ }^{10}$ de ce genos pénètre au prytanée, dont l'accès lui était formellement interdit, il doit être sacrifié. Contrairement à d'autres cas de sacrifices humains concernant certains peuples étrangers qu'Hérodote mentionne dans son œuvre, celui d'Alos est décrit plus en détail et met surtout en avant, comme le note bien R.Gagné, la procession sacrificielle, qui joue un rôle important dans ce rite, un rite qui implique toute la population et le territoire civique. L'auteur analyse, de façon avisée, ce rituel en 
portant l'attention sur la relation entre passé et présent, sur l'histoire d'Athamas, présentée comme "a tragic family drama", sur la place de la parenté dans la constitution de la cité et le rôle de l'oracle, sur l'expiation perpétuelle d'une faute qui pèse sur l'entière communauté, sur l'imbrication entre mythe et rituel, sur l'articulation entre « east and west in the Greek imagination ». Mais, l'intérêt de cette contribution se trouve aussi dans l'association établie par l'A. entre la narration d'Hérodote et l'invasion perse, menée par Xerxès contre la Grèce, une expédition placée sous le signe du sacrilège et de la transgression de l'ordre divin.

Avec l'érudition et la finesse des analyses qu'on lui connaît, Albert Henrichs revient à la question de la violence, inscrite, comme il dit, «dans l'identité des Grecs et de leur culture » («Répandre le sang sur l'autel : ritualisation de la violence dans le sacrifice grec $)^{11}$. Ce qui l'intéresse tout particulièrement, c'est la place et la fonction de la violence dans la religion grecque, la thématique du sacrifice animal et humain dans la tragédie, en prenant l'exemple d'Iphigénie, la mise en parallèle des victimes animales et humaines dans leur mise à mort rituelle, la question de savoir si les sacrifices d'animaux suscitaient chez les Grecs un sentiment de culpabilité. Ses remarques judicieuses sur une série de thèmes (les Grecs et la guerre, le rapport entre la guerre et la religion - deux domaines non séparés pour les Grecs -, le sang qui coule au combat, mais qui joue aussi un rôle essentiel « dans la réalité du rituel sacrificiel », le soi-disant "sacrifice animal volontaire", considéré par l'auteur comme "un euphémisme de la mentalité grecque » - et ce n'est qu'un choix parmi les sujets abordés) vont parfois à l'encontre de certaines idées préconçues ${ }^{12}$ et donnent de la vitalité au dialogue. A. Henrichs rejette avec raison l'explication habituelle qui renvoie l'origine des sacrifices humains "aux temps immémoriaux " et interprète certains rituels comme des "survivances" de ces immolations du passé. Il croit, comme d'autres spécialistes, que les « descriptions historiques de sacrifice humain... appartiennent invariablement au domaine de l'imagination et sont donc fictives" (une opinio communis qui, à mon sens, devrait être au moins nuancée). Mais, en même temps, l'A. pose la bonne question: il se demande "pourquoi le sacrifice humain demeura si longtemps et si fortement enraciné dans l'imaginaire des Grecs?». Finalement, par rapport à cet excellent article, je me permets d'exprimer une certaine réticence concernant, de nouveau, la définition du sacrifice humain comme « perversion du sacrifice animal [qui représenterait] la forme normale du culte ». Car je continue à penser que la théorie du "sacrifice perverti », qui fonctionnerait comme une "valeur d'inversion", comme un acte "anti-norme", est plutôt inadéquate - dans sa rigidité, son aspect binaire, voire exclusif - pour rendre compte d'un rituel si complexe, exigé souvent et imposé par des divinités grecques, qui ne semblent pas avoir la réputation d'être "perverses" ou "anormales" (voir aussi ci-dessous).

Il faut savoir gré à Robert Parker d'être revenu à cette théorie tenace sur la substitution, portée par tout un discours anthropologique (cf. les travaux de H. Hubert, M. Mauss, E.E. Evans-Pritchard, L. De Heusch, etc.), selon laquelle un animal est sacrifié à la place d'un être humain ("Substitution in Greek Sacrifice »). La question posée par R. Parker est de savoir si l'idée de la substitution est «incidental and occasional or fundamental» dans la pratique et la pensée grecques. Il distingue trois types de substitution. Selon le premier, le sacrifice humain est évité à la dernière minute et remplacé par un sacrifice animal (cf. Iphigénie). D'après le second, la divinité qui demande l'immolation d'un être humain accepte par la suite de recevoir sur son autel un animal. Pour ce cas, R. Parker renvoie, comme exemples, au culte de Dionysos 
Aigobolos, en Béotie ${ }^{13}$, et à celui de Dionysos « Man-Tearer » à Ténédos. Si la substitution est claire dans le culte de Dionysos Aigobolos, d'après le récit de Pausanias, je ne pense pas que ce soit le cas dans le sacrifice du veau nouveau-né à Ténédos, en l'honneur de Dionysos Anthroporrhaistês, une épiclèse qui ne saurait être traduite par "Man-Tearer " (voir ci-dessus, note 5). Quoi qu'il en soit, ces deux types de substitution sont considérés, avec raison, par l'auteur comme "not very commun ». Quant au troisième type, il concerne le sacrifice humain par rapport au rituel sacrificiel accompli avant la bataille, un rituel qui pose le problème de "l'interchangeabilité entre animal et humain ». R. Parker fait une série de remarques pertinentes, lorsqu'il observe, par exemple, que le mythe hésiodique sur la ruse de Prométhée, le seul récit relatif à "l'origine du sacrifice», ne dit rien concernant la substitution; ou quand il note que les mythes, même ceux qui se situent dans un passé lointain, se réfèrent habituellement aux sacrifices d'animaux, tandis que la nécessité d'immoler une victime humaine constitue « a terrible exception» (mais qui advient, je dirais, plus souvent qu'on ne le croit). Ainsi, tout laisse penser, selon l'heureuse conclusion de R. Parker, que l'idée de la substitution ne semble pas avoir occupé une place centrale dans la réflexion et la pratique sacrificielles des Grecs.

17 La Chine offre souvent un terrain de comparaison privilégié avec la civilisation des Grecs. On ne s'étonne donc pas de trouver, après les six articles qui s'interrogent, chacun avec sa manière propre, sur le sacrifice grec, deux contributions consacrées à la Chine. Dans la première (" Human Sacrifice and the Ritual of War in Early China »), Robin Yates souligne d'emblée une différence significative avec le monde grec: tandis que, pour la Chine ancienne, les données archéologiques relatives au sacrifice, aussi bien humain qu'animal, sont abondantes, on ne dispose, sur ce sujet, que peu de témoignages textuels ou iconographiques, contrairement aux sources écrites grecques, qui constituent une mine précieuse pour l'immolation d'êtres humains. On pourrait distinguer deux types de victimes humaines: celles offertes aux esprits, et celles enterrées avec le mort, mais il est question aussi de ce que l'auteur appelle «selfsacrifice ", à savoir des suicides commis en particulier par de jeunes femmes, non pas pour "sauver la patrie ", comme en Grèce, mais pour échapper à l'enlèvement, ou parce qu'elles étaient violées, ou par loyauté envers leurs époux décédés (mais il n'est pas clair pourquoi ces suicides sont appelés " auto-sacrifices »). Ce dernier cas renvoie à une question importante posée par l'auteur: ceux qu'on appelle «morts d'accompagnement», peuvent-ils être considérés comme exemples de sacrifice humain ? À ce sérieux problème de définition, R. Yates semble répondre positivement, mais la question reste, me semble-t-il, ouverte. Cependant, ce qui compte surtout pour l'auteur c'est la guerre, considérée, en Chine ancienne, comme un rituel, une forme de sacrifice humain. En analysant ainsi certains manuels militaires anciens, R. Yates donne des exemples explicatifs portant sur les multiples dimensions du sacrifice humain et sur des pratiques rituelles militaires, en montrant la connexion étroite entre guerre, rite et loi.

18 C'est aussi sur un texte étonnant de la Chine ancienne qu'attire l'attention la deuxième contribution, sous la plume de Griet Vankeerberghen («"Yellow Bird" and the Discourse of Retainer Sacrifice in China »). Un texte intitulé «L'Oiseau Jaune », qui fait partie des Odes, une collection canonique composée de 305 poèmes, et complétée vers 600 avant notre ère. Ce court poème, largement et longtemps connu des générations successives, traduit l'histoire et les valeurs de la dynastie Zhou et relate la mort sacrificielle de trois hommes, membres éminents ${ }^{14}$ du clan Ziju, pendant les funérailles 
du Seigneur Mu qui a régné sur Qin (659-621). Présentant les trois strophes de ce poème, correspondant aux trois victimes, G. Vankeerberghen examine sa réception à travers les époques, les interactions entre croyances, émotions, traditions et relations de pouvoir, mais aussi les critiques du Confucianisme contre le Seigneur Mu et ce rituel sacrificiel. Différentes lectures de ce poème mettent l'accent sur les thèmes de «l'acte volontaire ", du "suicide loyal », de l'héroïsme ou de la peur, de la distinction barbare/ civilisé, de la relation entre sacrifice humain "régulier", et ce qu'on appelle (en anglais) « retainer sacrifice ", à savoir la mise à mort de serviteurs, artisans, concubines etc. pendant les funérailles de rois ou de personnes de haut rang, membres de l'aristocratie - ce qui pose de nouveau le problème de la définition de ces mises à mort de diverses personnes (selon les époques et les cultures), dans le but d'accompagner le défunt dans l'au-delà. En conclusion, selon $G$. Vankeerberghen, ce poème devrait être analysé comme un drame rituel avec sa logique inhérente, il devrait être lu comme un texte qui évoque le "retainer sacrifice» pour des raisons commémoratives. Une commémoration qui, loin d'indiquer la séparation entre la population de Qin et son chef - selon une interprétation précédente - montre que la communauté dans son ensemble soutient cette pratique sacrificielle, malgré la souffrance et la valeur des hommes immolés.

De la Chine aux Aztèques, la route est longue, mais elle mérite d'être parcourue pour trouver un univers, certes, différent, mais riche en interrogations sur le sacrifice humain. Dans son article («La représentation des sacrifices humains par les Aztèques et les Espagnols : une image vaut mille mots»), Louise Paradis soulève une question essentielle, qui pourrait aussi être posée pour d'autres cultures : comment considérer un fait cultuel, une pratique religieuse, lorsqu'on a affaire à des sources différentes, avec des visées propres, et souvent marquées idéologiquement. En l'occurrence, comment comprendre les pratiques sacrificielles aztèques, en prenant en compte, d'une part, les documents des Aztèques et, de l'autre, les descriptions ethnocentriques des chroniqueurs espagnols. En effet, l'étude de certains documents picturaux préhispaniques (comme les codices Borgia, Borbonicus et Fejérváry-Mayer), mais aussi l'examen de l'architecture et de la sculpture aztèques, reliées au sacrifice humain et caractérisées par leur aspect public et ostentatoire, montrent, selon L. Paradis, que les représentations des pratiques sacrificielles aztèques revêtent le plus souvent un sens métaphorique. En revanche, les documents visuels espagnols ainsi que les récits de chroniqueurs et conquérants, qui reconstituent des rituels «impliquant des sacrifices ou autosacrifices humains ", relèvent davantage de la narration et deviennent de plus en plus explicites, au cours du Xvi $I^{e}$ siècle. On peut donc parler de deux discours sur le sacrifice humain, deux discours dont l'examen permet de mieux comprendre comment les Aztèques concevaient les pratiques sacrificielles qu'ils accomplissaient et qui s'inscrivaient dans le contexte économique, politique et idéologique de leur État, par rapport à l'image horrifiée que les Espagnols se faisaient de ces pratiques, dénoncées souvent comme "barbares et païennes », comme l'œuvre du diable. - Une fois encore, on voit bien comment on taxe de "barbarisme" ce qui ne nous appartient pas, en l'occurrence, les pratiques et coutumes de l'Autre, des pratiques qu'on ne connaît pas, ou dont on est incapable, comme le dit bien L. Paradis, de saisir la complexité en les replaçant " dans leur contexte politique et religieux ».

Avec la dernière contribution du volume, nous regagnons des rivages plus familiers aux antiquisants, mais cette fois non pas du côté des Grecs. L'étude de Bill Gladhill («The Poetics of Human sacrifice in Vergil's Aeneid ») nous amène en effet vers Rome, en nous 
plongeant dans l'univers poétique d'une grande épopée. La question que se pose l'auteur est de savoir ce que signifie le sacrifice humain dans l'économie de l'Énéide, et ce qu'il nous dit sur la Rome de Virgile. Tout d'abord, il passe en revue certains arguments et positions de Cicéron, de Lucrèce et de Sénèque, où il est question de la nature de fides et de pietas, et où les causes du sacrifice humain, exprimées « in terms of transgressed fides ", restent inchangées. Cette continuité suggère, selon l'auteur, que le sacrifice humain dans l'Énéide devrait dépendre d'une crise similaire de fides, ritualisée entièrement au Livre 12. Bill Gladhill soumet ensuite le poème à une analyse serrée, en examinant scrupuleusement la mise à mort de certains personnages (dont Orontès, Priam, Mago ou Turnus), et en démontrant que Virgile associe effectivement le sacrifice humain aux origines de Rome. Ainsi, «le dernier sacrifice humain » mentionné dans l'épopée, celui de Turnus tué par Énée, serait «le dernier acte de transgressions sacrificielles ", un acte qui aurait entraîné comme conséquence la création d'une réalité politique et cosmologique nouvelle, la fondation d'une cité et d'un empire qui incarnent cette réalité, ainsi que l'abolition du sacrifice humain en tant que force du chaos. - Cette contribution, dense, réfléchie, faisant preuve d'une connaissance approfondie de ce grand poème qu'est l'Énéide, pose cependant - au moins à mon sens - un vrai problème : toutes ces mises à mort y sont qualifiées de "sacrifices humains ", sans que l'on puisse comprendre les raisons de cette définition. Certes, on ne va pas ouvrir ici le débat sur cette question, fondamentale à mon avis, mais la question mérite, me semble-t-il, d'être posée. J'aimerais, pourtant, dire deux mots à propos de ce que l'auteur appelle le « sacrifice of Priam » (cf. p. 233, 235), tout en parlant aussi parfois de "slaughter of Priam » (cf. p. 231, 237). Dans la description de la mise à mort de Priam par Néoptolème (Énéide II, 535-558), citée et commentée par B. Gladhill (p. 231-232), il n'y a rien qui puisse nous faire penser à un sacrifice (par exemple, du point de vue du vocabulaire $)^{15}$. Le fait que Néoptolème ait traîné (traxit) Priam avec force vers les autels situe, certes, cet acte violent dans un espace rituel, mais il renvoie sans doute à la fameuse scène où Priam, qui avait trouvé refuge, comme un suppliant, à l'autel de Zeus Herkeios, dans son propre palais, est poursuivi et tué par Néoptolème (Ilias parva, fr. 16 Allen, ap. Pausanias X, 27, 2). Or, s'il arrive parfois qu'on tue des suppliants, bien qu'ils soient mis sous la protection de Zeus Hikesios, commettant ainsi un acte sacrilège, source d'agos et de grands malheurs pour la communauté, on ne saurait les "offrir en sacrifice"! Quelle divinité, d'ailleurs, accepterait une telle "offrande" impie? Les dieux se détourneraient plutôt avec horreur d'un tel acte qui irait à l'encontre de ce qui est permis aux hommes, selon les règles religieuses, de ce qui est hosion. Je dirais même que la mention des autels (altaria), dans ce passage de l'Énéide, rend encore plus horrible, encore plus inacceptable pour les dieux, la mise à mort ignoble du vieux roi de Troie.

Doté d'une bibliographie sélective, très utile, avec 17 planches illustrant certains sujets traités dans le volume, mais privé d'un Index, ce qui est regrettable, cet ouvrage collectif remplit bien son rôle : chaque contribution, en effet, donne à penser, pose des questions, ouvre des voies. La présence prépondérante du monde grec n'avait sans doute pas permis de prendre en compte d'autres cultures qui se prêtent bien à ce sujet, comme par exemple, les Phéniciens. Par ailleurs, on aurait aimé trouver un dialogue plus tangible entre les contributeurs, qui auraient pu mieux réagir, l'un par rapport à l'autre, pour que la discussion comparative entre les spécialistes de diverses cultures éclaire davantage ce thème complexe, parfois même insaisissable, que représente le sacrifice humain. Mais cela n'enlève rien à l'intérêt de cet ouvrage qui, grâce à la valeur des textes présentés, a fait un pas de plus dans la connaissance et la compréhension de 
ce rituel sacrificiel, en mettant surtout l'accent sur les représentations des immolations humaines.

\section{Le sacrifice humain : entre païens, chrétiens et les autres}

Les questions suscitées par le dernier article du volume collectif animé par Pierre Bonnechere et Renaud Gagné constituent une bonne transition pour s'engager dans l'ouvrage, également collectif, qu'ont dirigé et introduit Agnès Nagy et Francesca Prescendi. Car cet ouvrage pose d'emblée le problème de la définition de la catégorie de "sacrifice humain", ainsi que de l'utilisation de cette notion. Les deux éditrices remarquent à juste titre que, sous l'expression "sacrifice humain ", sont réunis "des actes rituels dont les différences sont parfois tout aussi marquées que les ressemblances ». Elles décident finalement de continuer à utiliser cette expression, car elle permet "d'engager le dialogue entre les spécialistes des divers domaines de l'histoire des religions, de l'archéologie, de l'anthropologie et de la sociologie ». Mais, dans une "perspective comparatiste", qu'elles revendiquent, A. Nagy et F. Prescendi optent pour une définition large de "sacrifice humain », qui comprend aussi ce qu'elles appellent « meurtres rituels » et, en général, « des rites visant à honorer un être ou une entité surhumains, mais aussi ceux qui ont pour but d'assurer la sauvegarde, le bonheur et la prospérité d'une personne ou d'une entité supérieure à l'individu comme le roi, la communauté, la patrie, etc.». Car cette définition large pourrait «s'adapter à des contextes culturels différents, du passé et du présent ». Cette position n'est pas sans soulever quelques réserves, ou susciter quelques réflexions et remarques, qu'on ne saurait développer ici. Relevons cependant certains points soulignés en particulier par les deux éditrices dans leur Introduction. Tout d'abord, elles notent avec raison que le sacrifice humain n'est pas " un simple équivalent du sacrifice animal », car il y a, entre autres, une différence significative qui les sépare : la victime animale peut servir de repas aussi bien aux dieux qu'aux participants, contrairement à la victime humaine dont le sacrifice - pour rappeler les paroles du Chœur dans l'Agamemnon d'Eschyle, 150 - est qualifié d'adaitos, "sans repas », " sans festin ». Ensuite, elles mettent l'accent sur «le jugement de valeur exprimé par les Grecs et les Romains sur les sacrifices humains » (critiques de certains philosophes, ou de courants philosophiques etc.), sur la présentation du sacrifice humain "par les auteurs anciens " comme « un rituel de l'Autre » et "en même temps comme un rite du passé » ${ }^{16}$, et finalement sur le fait que l'interdiction du sacrifice humain imposée par toute une série de mesures décrétées par les Romains sur tout le territoire (comme, par exemple, le sénatus-consulte de 97 avant notre ère), montrerait que la fin de cette pratique précède l'avènement du christianisme qui, de toute façon, va rejeter le sacrifice humain « du côté des erreurs monstrueuses du paganisme».

Cet ouvrage est judicieusement divisé en cinq parties, qu'on essaiera de suivre de façon succincte, en laissant obligatoirement de côté des aspects importants qui mériteraient un développement ultérieur. 


\section{Question de définition}

24 des a priori : quelques remarques méthodologiques »), attire justement l'attention sur la difficulté de concevoir le discours des différentes sociétés sur le sacrifice humain d'après les sources disponibles, qui ne sont pas toujours de la même nature : littéraires, iconographiques, archéologiques pour certaines sociétés anciennes (comme la Grèce et Rome); archéologiques et parfois iconographiques pour d'autres cultures (comme les Maché du Pérou), pour lesquelles on ne dispose pas des textes écrits ; seulement orales pour d'autres populations, dont les valeurs sont transmises par le discours des ethnologues. Du point de vue de la définition, $\mathrm{P}$. Bonnechere réaffirme sa préférence pour maintenir le terme de "sacrifice humain", en lui donnant un sens large, englobant "toute mort rituelle d'un être humain dans un contexte religieux", ou «magico-religieux, voire à tendance religieuse »; il considère ainsi qu'une définition trop stricte, qu'il qualifie de "maurassienne " (à savoir, "l'offrande d'une victime humaine à un destinataire suprahumain»), aurait éliminé " une part importante du rituel ». Reconnaissant les difficultés et les apories du comparatisme, l'auteur s'élève avec raison contre les présupposés évolutionnistes, ou «l'imposition d'un modèle propre à une culture à toutes les cultures du monde ». Contre un "schéma unique », il faut "admettre des voies multiples" - une conclusion à laquelle on ne peut qu'exprimer son entière adhésion. Mais je suis plus sceptique lorsqu'il parle de la " condamnation unanime » du sacrifice humain "d'Homère à l'époque romaine », ou bien quand il affirme que "le schéma de substitution des victimes, humaines puis animales, est très répandu, en Grèce et à Rome ${ }^{17}$, ou encore lorsqu'il adopte la thèse de Karl Meuli suivi par Walter Burkert ( le sacrifice animal est l'héritier des chasses paléolithiques ritualisées lors du néolithique $»^{18}$ ), pour montrer que, s'il en est ainsi, le sacrifice humain n'a pas pu être "antérieur au sacrifice animal ». Cependant, au-delà de ces positions, l'intérêt du texte de P. Bonnechere se trouve surtout dans la comparaison méthodologique qu'il établit entre Grecs et Aztèques, qui montre, entre autres, qu'un "comparatisme éclairé» doit porter non tellement sur «les faits proprement dits », mais sur « la façon d'interroger les documents ».

La définition large du "sacrifice humain ", défendue, dès le début, dans cet ouvrage, ne convient pas, semble-t-il, à tout le monde. Dans son article suggestif ( « Observations sur l'anthropoctonie. Le débat sur les "sacrifices humains" en Égypte ancienne »), Youri Volokhine, tout en se référant aux deux orientations distinctes du sacrifice humain (" étroite " et "élargie »), met en question la catégorie du «sacrifice humain », qu'il souhaite replacer dans le cadre plus général de la notion du "sacrifice». Car on devrait d'abord repenser et redéfinir cette notion « dans le champ de la culture de l'Égypte pharaonique ». Or, si l'offrande est fondamentale «dans les processus rituels égyptiens, en revanche, l'acte amenant à cette offrande ", en l'occurrence les mises à mort (d'animaux, par exemple) ne semblent pas être considérées comme des "actes centraux » du culte (une remarque qu'on pourrait faire aussi pour le sacrifice sanglant grec). Au lieu donc de parler de "sacrifice humain", l'auteur opte pour la catégorie d'« anthropoctonie ", dans le sens de " mise à mort d'êtres humains lors de procédures ritualisées ». Il constate que le « motif de l'Égyptien anthropoctone » se constitue selon une double tradition, grecque et moderne, et s'inscrit dans «le cadre de la pensée occidentale». Il explore ainsi, d'une part, le discours grec sur le sacrifice humain égyptien et, d'autre part, les interprétations modernes des données archéologiques. Il 
observe, par ailleurs, que la représentation de la mise à mort de personnes considérées comme des ennemis du pays et des dieux égyptiens n'implique pas "la performance effective ». Le motif iconographique du roi en train de massacrer les ennemis agit plutôt par «virtualité symbolique»; l'exercice du pouvoir passe en effet par «l'exaltation du massacre et de l'écrasement de l'adversaire ». L'Égypte ancienne a donc pensé la mise à mort d'êtres humains, mais, en réalité, elle l'a pratiquée «moins qu'elle ne l'affiche».

Pour clore cette première partie, Agnès Nagy choisit, dans son article («L'ordalie "primitive" entre sacrifice humain et peine de mort: sur les traces d'un mythe savant»), de s'interroger sur ce qu'elle considère comme un concept du sacrifice humain, à savoir l'ordalie "primitive " ou "originelle», un concept qui touche à l'histoire du droit, à l'histoire des religions, à l'anthropologie. Elle se lance donc dans une enquête historiographique, scrupuleuse et bien utile, qui va du Haut Moyen Âge à nos jours, en passant par les historiens du XIX ${ }^{e}$ siècle et ses courants évolutionnistes. Selon la majorité des auteurs du $\mathrm{xvIII}^{\mathrm{e}}$ siècle, l'ordalie, considérée comme illégitime, comme œuvre des démons, n'a «aucun lien direct avec le sacrifice humain». En revanche, pour celui qui est vu comme le " père » de la conception moderne de l'ordalie " primitive ", à savoir Gustave Glotz ${ }^{19}$, et à qui A. Nagy prête une attention particulière, l'ordalie, une institution dont les traces se trouvent dans la "Grèce primitive ", ne fonctionne pas seulement comme une forme de divination: elle est également un procédé « magico-religieux » d'une époque reculée, et étroitement associée au sacrifice humain considéré, lui aussi, comme « signe de primitivisme ».

\section{Sacrifice humain vs sacrifice animal}

La relation entre sacrifice animal et sacrifice humain constitue une thématique récurrente dans le discours des historiens des religions. Mais elle peut aussi être sujet à réflexion pour un auteur savant et polyvalent, comme le théologien zurichois Stuchi, auquel Marc Kolakowski consacre sa contribution («Humana, seu potius inhumana sacrificia. Le sacrifice humain à la croisée des discours dans l'œuvre du polyhistor Johann Wilhelm Stucki (1542-1607)»). Connu surtout pour son traité sur les mœurs de table, ce Suisse réformateur écrit une "Brève et soigneuse description des cultes et sacrifices des Gentils " (Sacrorum sacrificiorumque gentilium brevis et accurata descriptio), publiée à Zürich en 1598. S'appuyant tant sur les auteurs classiques que sur les Pères de l'Église, Stucki pose déjà des questions pertinentes sur le temps du sacrifice, les destinataires, les objets, les lieux, les agents, les étapes, les fins. Il inscrit le sacrifice humain dans les "objets ou matériaux", c'est-à-dire dans les "offrandes sacrificielles », qu'il divise en trois catégories : "animées ", « inanimées ", « mixtes ». Or, la première catégorie est subdivisée en deux sous-catégories : celle des êtres « doués de raison", auxquels appartient la victime humaine, et celle des êtres "privés de raison ", dont font partie les animaux, surtout les bœufs, les victimes animales par excellence. Il y aurait donc une possible confusion entre l'homme et l'animal, puisque, dans la pratique du sacrifice humain l'homme serait traité "comme un bœuf", seulement comme un corps privé de son âme. Et à côté de cette confusion homme/bête et de la "violence faite au corps ", on peut entrevoir chez Stucki - comme l'affirme Marc Kolakowski - une confusion humain/inhumain : le sacrifice humain devient aussi "inhumain " à cause de celui qui l'exécute, qui accomplit ou suscite l'immolation d'êtres humains, une inhumanité cruelle, derrière laquelle, Stucki, en bon théologien, 
voit la main du diable. Ainsi, à l'erreur et la confusion diaboliques des Gentils qui offrent leurs sacrifices «(in)humains » à de nombreuses divinités, Stucki oppose le vrai sacrifice « humain», celui des chrétiens, qui s'adresse à un Dieu unique, «le seul vrai dieu ».

Comme la Chine, l'Inde offre un bon terrain de comparaison avec d'autres cultures. Ce n'est pas dans cette optique que se situe l'article de Johannes Bronkhorst («Des sacrifices humains dans l'Inde ancienne »), ce qui ne veut nullement dire que ses réflexions n'offriront pas matière à penser aux spécialistes d'autres religions. Ce qui l'intéresse surtout, ce sont, tout d'abord, les deux types de sacrifice humain dans la littérature brahmanique ancienne : dans le premier cas, c'est le sacrificateur lui-même qui se sacrifie, en se jetant dans le feu sacrificiel; dans le deuxième, c'est une victime humaine mâle, appartenant à l'une des deux classes supérieures ${ }^{20}$, qui est achetée à sa famille et gardée pendant un $\mathrm{an}^{21}$, avant d'être mise à mort. Ces deux types de sacrifice ne sont pas fréquents dans la littérature védique, où l'on trouve plutôt l'idée de la substitution. Au lieu de "s'autodétruire " (nous dirions, ailleurs, de "s'autosacrifier "), le sacrificateur met à mort un substitut de lui-même. Mais comme le remarque l'auteur, les deux types de sacrifices se mélangent souvent, comme pourrait le montrer le sacrifice du cheval, considéré comme un grand sacrifice royal védique : le roi laisse errer librement un cheval, pendant presque un an ${ }^{22}$, avant de le sacrifier, dans le cadre d'un rituel qui suggère que le cheval est considéré comme le substitut du roi. Finalement, pour J. Bronkhorst, « il n'y a, au fond, que des sacrifices humains », dont certains « mettent un substitut à la place de l'être humain » - ce qui montre sans doute que, contrairement à la Grèce, l'idée de la substitution occupe une place importante dans la pratique sacrificielle indienne. J'aimerais cependant souligner une question essentielle que pose, à la fin, l'auteur, en se demandant où sont les dieux dans tout cela. Or, il constate que, dans le sacrifice indien, leur rôle "peut être faible, voire inexistant ", une réponse qui va, cette fois aussi, à l'encontre de la situation grecque, où les dieux sont bien présents dans le sacrifice humain.

\section{Dossiers archéologiques}

29 La contribution de Gilbert Kaenel est, à mon avis, doublement appréciable (« Gaulois et sacrifices humains: des textes antiques aux observations archéologiques»). Tout d'abord, car elle envisage clairement le sacrifice humain comme une pratique qui a un destinataire, qui s'adresse à une "entité supérieure", en général à une divinité. Et ensuite, car elle met en garde contre la difficulté, pour un archéologue, de distinguer les raisons de la mise à mort d'un individu dont il examine les os : s'agit-il d'un sacrifice humain, d'un acte donc religieux, ou bien d'une action judiciaire, "d'un châtiment infligé à des justiciables », ou encore "d'un fait de guerre? ». Dès lors, si l'existence du sacrifice humain chez les Gaulois, "comme dans la plupart des sociétés antiques de Méditerranée ", ne fait aucun doute, s'il est possible que certains individus aient pu faire « l'objet d'un sacrifice dans le monde gaulois de la seconde moitié du I ${ }^{\mathrm{er}}$ millénaire avant notre ère ", on ne peut pas le démontrer, le prouver, "à l'aide des seules méthodes de l'archéologie ».

En explorant la culture Mochica ou Moché, développée «sur la côte nord du Pérou, entre les $\mathrm{II}^{\mathrm{e}}$ et VIII ${ }^{\mathrm{e}}$ siècles de notre ère ", Steve Bourget ("Sacrifice, violence rituelle et développement de l'État Mochica dans le Pérou ancien ») reconnaît qu'on manque « de 
preuves tangibles de sacrifices humains", de données détaillées, pour qu'on puisse faire une étude approfondie d'une des "pratiques rituelles les plus complexes", associées au religieux, au politique, au social. Il s'efforce, néanmoins, de trouver quelques indices de ce rituel, grâce à certaines tombes « de dirigeants et d'officiants religieux ", découvertes à partir des années 1980. Selon l'auteur, l'examen de ces tombes, ainsi que la comparaison entre les objets retrouvés dans ces sépultures et l'art figuratif mochica, donnent des éléments susceptibles de prouver la pratique du sacrifice humain chez les Mochica. Une pratique associée étroitement au pouvoir, comme il ressort de l'enquête conjointe des données archéologiques et iconographiques, une enquête qui fait particulièrement référence à la violence rituelle, à l'usage du sang, au thème de la guerre rituelle et de la capture d'individus destinés au sacrifice. - Cependant, bien qu'il soit souvent question d'activités et de victimes sacrificielles, on voit moins clairement à qui s'adressent ces actes, quel est leur destinataire, sauf si l'on fait, avec l'auteur, l'hypothèse que les individus à qui l'on offre «le sang des victimes» sur certaines images sont peut-être de nature divine, une supposition dont la vérification incombe, évidemment, aux spécialistes du Pérou ancien.

31 C'est sur une grande découverte archéologique et ses multiples problèmes que se penche Anne-Caroline Rendu Loisel dans sa contribution « Le cimetière royal d'Ur : état de la question ». Car ce cimetière $\mathrm{e}^{23}$, mis au jour au début $\mathrm{du} \mathrm{xx}^{\mathrm{e}}$ siècle, a été considéré par certains comme la preuve de la pratique du sacrifice humain en Mésopotamie ancienne, bien que cette pratique soit presque absente des sources cunéiformes ultérieures. Il s'agit souvent de tombes richement équipées, où sont enterrés des personnages de haut rang, accompagnés d'autres personnes, sans doute des membres de leur suite, inhumées simultanément. L'auteure propose donc une enquête historiographique et chronologique des différentes théories élaborées par les spécialistes, une recherche qu'elle mène minutieusement, en présentant les diverses hypothèses, interprétations ou thèmes qu'inspire cette étonnante découverte. On a parlé, entre autres : de tombes de rois et de reines d'Ur, divinisés de leur vivant et enterrés avec leur cour; d'un rite de mariage sacré; d'une preuve de la présence d'envahisseurs (Scythes); d'une pratique de substitution pour apaiser la colère divine ; d'inhumation des membres du clergé d'Ur; de rituels funéraires d'accompagnement mettant l'accent sur le pouvoir des souverains de ces époques, etc. De ce foisonnement de propositions, il faut, me semble-t-il, mettre en avant le thème de ce qu'on appelle "morts d'accompagnement", attestés dans de nombreuses cultures et étudiés particulièrement par l'anthropologue Alain Testart ${ }^{24}$. Car, selon Testart, il ne s'agirait pas, dans ce cas, de "sacrifice humain ", comme on le prétend souvent, mais d'une pratique sociale qui témoigne de la dépendance, de la fidélité, de la hiérarchie de personnes et de fonctions.

\section{Sacrifice humain et christianisme}

C'est une heureuse idée de prendre en compte, dans un ouvrage collectif consacré au sacrifice humain, les positions chrétiennes sur ce rituel. Car si la comparaison entre différentes cultures polythéistes est nécessaire et enrichissante, il est tout aussi indispensable de scruter et appréhender les réactions d'une religion monothéiste comme le christianisme. C'est Simon Mimouni qui ouvre cette partie ( La tradition du dernier repas de Jésus au $\mathrm{I}^{\mathrm{er}}$ siècle : de la réalité historique à la réalité liturgique »), 
pour présenter le point de vue des chrétiens du $\mathrm{I}^{\mathrm{er}}$ siècle, en s'appuyant sur une lettre de Paul de Tarse datée des années cinquante, une source estimable, puisqu'il s'agit du plus ancien texte relatif au dernier repas de Jésus et de ses disciples. S. Mimouni soumet ce texte à une analyse littéraire et historique serrée, et fait des remarques et des constatations significatives, qui bousculent certaines idées reçues et donnent à penser à des spécialistes d'autres religions. Par exemple - et cela malgré les apparences - la tradition de ce dernier repas de Jésus «n'entretient aucun rapport avec les sacrifices, encore moins avec les sacrifices humains ». Il s'agit plutôt d'un " acte de partage qui est ensuite érigé en rituel», un acte qui renvoie à des repas communautaires ou associatifs, comme ceux qu'on voit dans le monde gréco-romain. On ne devrait donc pas confondre la tradition du dernier repas avec l'institution de l'eucharistie, dont le caractère sacrificiel n'apparait pas avant 70 et la destruction du Temple. C'est donc plus tard, au II $^{\mathrm{e}}$ siècle, que l'eucharistie est interprétée comme l'équivalent d'un sacrifice, d'un sacrifice "éternel et universel ", d'une "efficacité infinie et inépuisable ». Mais, auparavant, pour les chrétiens qui vivent dans le contexte du judaïsme de cette époque, les sacrifices ne s'accomplissent que dans le(s) sanctuaire(s). Certes, comme le remarque l'auteur lui-même, cette contribution ne s'accorde pas bien avec le sujet central de ce volume, consacré aux sacrifices humains. Cependant, tout en se développant à la marge de cette thématique, l'article de S. Mimouni est particulièrement intéressant pour un helléniste; car il met, entre autres, en valeur deux réalités cultuelles (mais aussi culturelles) qui, dans la littérature chrétienne en général, sont désignées par deux termes grecs, dont le sens évolue et se

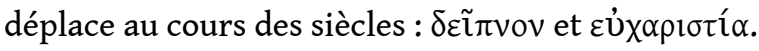

Ce ne sont pas seulement des chrétiens qui accusaient les païens des pires crimes et abominations. Il y a aussi des accusations de cannibalisme et de sacrifice humain lancées en sens inverse, contre les chrétiens, et c'est justement cette direction qui intéresse Jan Bremmer («Early Christian Human Sacrifice, between Fact and Fiction »), qui pose une série de questions : sur les dates, les lieux et les personnes impliquées dans ces accusations; sur la façon dont les modernes (à commencer par Gibbon) ont réagi et réagissent à ces accusations; sur les raisons de cette campagne diffamatoire contre les chrétiens. Son enquête fait appel aux apologistes de langue grecque et latine, comme Justin et son disciple Tatien, Athénagoras, Tertullien, Minucius Felix etc., qui contre-attaquent parfois en se référant à des actes cannibales des Grecs (Pélops, Kronos et les autres). Par ailleurs, les chrétiens étaient également accusés d'inceste, d'homosexualité (selon l'apologiste Aristide, à l'époque de l'empereur Hadrien), mais aussi d'" athéisme", d'après Tatien qui rapporte même que les païens appelaient les chrétiens atheotatous (« les plus athées »). En discutant la réception de ces accusations de la part de certains chercheurs, J. Bremmer rejette la thèse que ces accusations étaient inventées par des chrétiens "orthodoxes" pour diffamer et discréditer des chrétiens « hérétiques ». Quant aux raisons, l'auteur met en avant la façon dont nombre de païens percevaient les chrétiens, à savoir comme des gens très différents d'eux, refusant les normes religieuses en vigueur, voire comme une menace et un danger pour le monde gréco-romain.

L'histoire du roi de Suède Aun, que les lecteurs de Fraser ont peut-être rencontré dans le fameux Golden Bough, a été choisie par Bruce Lincoln ("King Aun and the Witches »), sans doute parce qu'elle pose un problème de pouvoir et de son abus. La meilleure version de cette histoire se trouve dans un texte composé entre 1220 et 1235, et dont la 
compilation est attribuée à Snorri Sturluson (1178-1241). Bien que considéré, dans ce texte, comme "a wise man and great sacrificer, but not a warrior", Aun se comporte comme un prédateur contre ses propres enfants, afin de se maintenir au trône et de prolonger sa vie au-delà des limites humaines. Pendant de longues années, et par intervalles, il sacrifie successivement à Odin neuf de ses dix fils et gagne ainsi quelques années de vie. Mais lorsqu'il a voulu sacrifier aussi le dixième fils pour continuer à vivre, il a été empêché par les Suédois, ce qui a provoqué sa mort. Pour la compréhension de cette histoire et de ses diverses implications et ramifications, B. Lincoln évoque, entre autres, certaines histoires similaires, comme, par exemple, le mythe grec de Kronos ou les légendes indiennes du Roi Yayāti, et conclut sur un constat : bien qu'on ait sûrement affaire à des cas de sacrifices humains réels, accomplis sous certaines conditions, "in most parts of the world and most periods of history, discourse about human sacrifice is more prevalent, more significant, and more revealing than its actual physical practice ». Une conclusion qui exalte la force que peut avoir le discours, son impact sur les esprits et les mentalités, à travers les âges.

Pour clore cette $\mathrm{IV}^{\mathrm{e}}$ partie, Nicolas Meylan nous amène vers l'Islande et la métropole norvégienne aux XII ${ }^{\mathrm{e}}$ et $\mathrm{XIII}^{\mathrm{e}}$ siècles ("Sacrifice humain et Islande républicaine, le cas d'óláfr Tryggvason »). Si l'on en croit la littérature islandaise du xIII siècle, les sacrifices humains étaient bien une réalité dans la Scandinavie préchrétienne. Deux récits, analysés par l'auteur, témoignent surtout de cette réalité, mais dans des buts différents. Le premier, transmis en Islande par deux textes (Gautreks saga et Gesta Danorum), raconte la mise à mort du roi païen Víkarr, accomplie par la fraude et la tromperie, pendant une expédition maritime, où, à cause des vents contraires, l'armée reste immobilisée. La comparaison avec l'histoire d'Iphigénie à Aulis est étonnante. Car, dans des conditions semblables, c'est également ici une divinité, le dieu Odin, qui exige un sacrifice humain, c'est à lui qu'on « donne » la victime. Mais la comparaison s'arrête là ; car le roi est tué par pendaison, et aucun animal ne vient remplacer la victime sur un autel qui n'existe pas. Le deuxième récit, dû à l'Islandais Snorri Sturluson (voir cidessus, l'article de B. Lincoln), met en scène le « roi missionnaire óláfr Tryggvason, qui régna sur la Norvège entre 995 et l'an mil ", et à qui on attribue la conversion de l'Islande. Or, cette fois, c'est ce roi chrétien qui organise " un grand banquet », et fait faire "le plus grand sacrifice qu'on connaisse", en sacrifiant des hommes. Selon N. Meylan, le premier récit s'inscrit clairement dans la volonté de dénigrer les païens, en considérant les sacrifices humains comme l'apanage de la période préchrétienne. En revanche, l'interprétation du second récit semble plus compliquée, car il présente le chrétien óláfr Tryggvason comme un personnage violent et dangereux, "plus païen que les païens ", puisqu'il a pris l'initiative de sacrifier des humains, pendant le repas communautaire des paysans qui, eux, n'abattaient que des moutons, des chèvres et des chevaux. Pour N. Meylan, le texte de Snorri, loin d'être un témoignage sur les sacrifices humains dans la Scandinavie médiévale, a une visée politique: il discrédite la monarchie norvégienne et son roi, qui veut annexer l'Islande à la Norvège, tout en illustrant les excès et l'arbitraire d'un pouvoir royal étranger qui menace l'Islande « républicaine ».

\section{De l'historiographie à l'imagerie culturelle}

Grâce à la contribution de Sergio Ribichini («Histoires de Moloch, le roi effroyable »), on prend enfin contact avec la Phénicie et Carthage, des terrains si prometteurs pour le 
thème du sacrifice humain. En partant des trois témoignages grecs (Clitarque, Diodore, Plutarque) qui parlent d'une statue de bronze de Kronos, au-dessus d'un brasier où l'on brûle des enfants qu'on lui sacrifie, chez les Phéniciens et surtout les Carthaginois, l'auteur retrace la passionnante histoire de cet «horrible » roi Moloch, «aspergé du sang des sacrifices humains et des larmes des pères et des mères ", selon l'image que brosse John Milton dans Le Paradis perdu (1667). Il montre comment se constitua une littérature "historique et exégétique », qui puise dans les traditions classiques et les textes bibliques, en les arrangeant, pour « appliquer aux rites sanglants de la Palestine préclassique les informations littéraires sur le sacrifice humain en milieu phénicopunique", et pour "donner nom et personnalité cannibalesque au monstrueux Moloch-Baal-Kronos-Saturne ». Par ailleurs l'image de cet idole avide d'enfants, mais aussi de vierges, n'a pas manqué d'inspirer, au fil du temps, des romans (cf. Salammbô de Gustave Flaubert), des œuvres d'art, des films, des bandes dessinées, voire des jeux vidéo, en fonctionnant, entre autres, "comme allégorie d'un âge et d'une croyance primitifs ", en donnant de Carthage une "vision négative », en déformant et adaptant Moloch aux exigences et aux idéologies d'une époque donnée. Face à cette déferlante d'images et de discours négatifs, l'archéologie vient combattre les présupposés et les préjugés idéologiques, et proposer d'autres interprétations, même s'il n'y a pas accord entre les spécialistes. En effet, les fouilles des sanctuaires appelés tophets, en Tunisie, Algérie, Sicile et Sardaigne, ont mis au jour des inscriptions votives (et non funéraires), ainsi que des urnes avec des restes incinérés de jeunes enfants, mais surtout de " fœtus, de mort-nés, ou de nourrissons décédés quelques jours ou semaines après leur naissance " (comme le montre le tophet de Carthage). Ce qui pourrait faire penser entre autres explications - à une consécration aux dieux des enfants morts précocement, " pour accomplir un vœu, pour invoquer l'aide divine pour soi-même et pour le salut des enfants, pour demander une nouvelle progéniture» etc. On est loin d'enfants brûlés devant la monstrueuse idole de Moloch, en laissant leur dernier souffle dans le fameux rire « sardonique »!

Avec son savoir sacrificiel indiscutable, Francesca Prescendi ouvre le dossier du roi éphémère des Saturnales, destiné à être sacrifié à la fin d'une période festive («Du sacrifice du roi des Saturnales à l'exécution de Jésus »). Un des points importants de son enquête est la discussion entre savants (en particulier entre James Georges Frazer et Franz Cumont) sur la mention d'un sacrifice humain dans un manuscrit grec (découvert par Cumont et daté probablement $\mathrm{du} \mathrm{x}^{\mathrm{e}}$ ou du $\mathrm{xI}^{\mathrm{e}}$ siècle), un texte qui relate le martyre du Saint Dasius. Le débat porte sur l'authenticité de cette mention (rejetée par Cumont, soutenue par d'autres), sur la comparaison étroite entre la passion du Christ et le traitement infligé, d'une part, au roi des Saturnales et, d'autre part, au roi de la fête babylonienne des Sacées, dont la fête juive de Purim serait, selon Frazer, une survivance. F. Prescendi critique, avec beaucoup de justesse, les théories frazériennes sur l'histoire en trois étapes du sacrifice humain, qui dénotent un évolutionnisme des coutumes et de la société qui seraient passées de la barbarie à la civilisation. Ainsi, chez les peuples "civilisés", le sacrifice humain primordial des anciens temps aurait été remplacé par des formes plus évoluées, par des " exécutions rituelles et symboliques »: la pratique de cette "relique" barbare n'aurait donc lieu qu'occasionnellement, «à des moments spécifiques de l'histoire des différents peuples ». Mais ces théories, remarque l'auteure, présentées déjà dans l'Antiquité, continuent à occuper les esprits; en particulier, «la mort rituelle du roi » a inspiré des savants, comme Lévi-Strauss, mais 
aussi des romans ou des pièces théâtrales, dont le Bacchus de Jean Cocteau est un exemple caractéristique de ces croyances à la "survivance" des rites anciens.

Enfin, comme un épilogue à cette cinquième partie, Aurore Schwab analyse certains rapports des Nations Unies concernant les crimes d'honneur, dans une perspective, comme elle dit, «d'histoire des religions» («La pratique du crime d'honneur: entre mythe et réalité »). Inscrit au sein de la famille aussi bien que de la communauté, le crime d'honneur est présenté également comme une pratique rituelle, "une forme ritualisée de violence » où les auteurs sont habituellement des hommes, tandis que les victimes sont des femmes. Réfléchissant sur la façon dont certains membres onusiens appréhendent les crimes d'honneur, A. Schwab pose la question de la relation entre magie, religion et raison, dans une perspective évolutionniste associée à l'idéologie du progrès. Sa conclusion montre la difficulté qu'implique un rapprochement supposé entre le crime d'honneur et le sacrifice humain. Car, comme elle le constate par ellemême, "au niveau terminologique, il n'est jamais question de sacrifice» et, par ailleurs, la femme tuée "ne semble guère être considérée comme un don à une divinité ». Elle préfère donc, plus prudemment, parler de «mise à mort rituelle ou de meurtre ritualisé ». - On le voit bien : il ne suffit pas qu'une pratique soit estampillée "religieuse", pour que la mise à mort d'un être humain soit qualifiée de "sacrifice humain".

On arrive ainsi à la fin de cet ouvrage collectif, consacré aussi, comme le précédent, au thème du sacrifice humain, et publié la même année, ce qui montre un intérêt renouvelé et réactivé depuis quelques années pour un sujet qui touche plusieurs cultures, qu'elles soient voisines ou éloignées dans le temps et l'espace. Dans le cas aussi de cet ouvrage, chaque contribution capte l'intérêt du lecteur, par sa qualité, par les questions qu'elle pose, par ses approches, par les ères culturelles qu'elle concerne. La division du volume en cinq parties thématiques rend la lecture plus systématique et facilite la compréhension. On fait aussi un bon usage des images, là où elles sont indispensables pour mieux saisir les faits exposés et suivre l'argumentation (articles de G. Kaenel et de S. Bourget). Pour couronner l'ouvrage, les Réflexions conclusives de Guy Stoumsa mettent l'accent sur la difficulté de cerner le sacrifice humain, sur «l'horreur sacrée » qu'il inspire "toujours et partout », mais aussi sur son utilisation moderne comme métaphore, ce qui expliquerait qu'il reste encore présent dans nos cultures. Enfin, j'aimerais tout juste signaler de nouveau l'avantage qu'aurait représenté un Index puisqu'il aurait permis une communication plus aisée entre les articles, une meilleure perception des « mots et des choses ».

Ces trois ouvrages, dont on a essayé d'esquisser ici le profil, sont, sans aucun doute, des outils indispensables pour tous ceux qui s'intéressent ou qui vont s'intéresser aux diverses facettes du sacrifice humain, en s'aventurant dans diverses cultures et contextes religieux, d'un bout du monde à l'autre, des temps anciens à l'époque contemporaine. Mais ils regorgent tellement de faits, d'idées, de questions, d'interprétations, qu'il est impossible, voire exclu, d'aller plus loin ici dans la discussion, par manque aussi de compétence dans des domaines pas très familiers aux hellénistes. Cependant, je me permets de conclure cette revue des livres par deux mots, en signalant tout simplement, sans entrer dans aucun débat, quelques points relevés ici et qui continuent de me poser problème, en espérant qu'on pourra reprendre plus tard cette discussion passionnante, comme le mérite un tel sujet. Ces points concernent 
surtout : le sens « large » ou « élargi » qu'on donne souvent à l'expression de « sacrifice humain ", en englobant dans cette catégorie démesurée toutes sortes de mises à mort et de meurtres; l'aspect "anti-norme» qui aurait caractérisé cette pratique, considérée comme " contraire aux règles » d'une société - une thèse qui devrait, à mon sens, être beaucoup plus nuancée; la place importante qu'occupent les dieux dans le sacrifice humain - très peu présents dans les deux ouvrages collectifs - par rapport aussi au vocabulaire sacrificiel, qui n'est pas toujours pris en compte comme il devrait; l'affirmation, souvent exprimée et bien résumée ici par G. Stroumsa, que "chez les Grecs, les Hébreux, les Romains, le sacrifice humain signalait la limite entre civilisation et barbarie ", une thèse qui, au moins pour les Grecs, reste très vague et imprécise. On n'ira pas plus loin dans cette brève énumération des points problématiques qu'on affronte lorsqu'on se plonge dans un sujet dont on ne se débarrasse pas si facilement, un sujet si décrié, mais qui continue à intriguer et à captiver les esprits.

\section{NOTES}

1. Pierre BONNECHERE, Renaud GAGNÉ (éd.), Sacrifices humains. Perspectives croisées et représentations / Human sacrifice. Cross-cultural perspectives and representations, Liège, Presses Universitaires de Liège, 2013. 1 vol. $16 \times 24$ cm, 266 p., 16 pl. (Religions. Comparatisme - Histoire - Anthropologie, 2). ISBN : 978-2-87562-021-7 ; Agnès A. NAGY, Francesca PRESCENDI, Sacrifices humains. Dossiers, discours, comparaisons, Actes du colloque de Genève, 19-20 mai 2011, Turnhout, Brepols, 2013. 1 vol. $15,5 \times 23,5 \mathrm{~cm}, 280$ p. (Bibliothèque de l'École des Hautes Études, Sciences religieuses, 160). ISBN : 978-2-503-54802-8.

2. Agnès A. NAGY, Qui a peur du cannibale? Récits antiques d'anthropophages aux frontières de l'humanité, Turnhout, Brepols, 2009. 1 vol. 15,5 × 23,5 cm, 280 p. (Bibliothèque de l'École des Hautes É tudes, Sciences religieuses, 140). ISBN : 978-2-503-53173-1.

3. Voir son ouvrage de référence: Le Sacrifice humain en Grèce ancienne, Athènes/Liège, 1994 (Kernos, suppl. 3).

4. F. PRESCENDI, Décrire et comprendre le sacrifice. Les réflexions des Romains sur leur propre religion à partir de la littérature antiquaire, Stuttgart, 2007, surtout p. 169-202.

5. En revanche, A. Nagy passe parfois un peu vite sur certains textes grecs, ou elle fait trop confiance à certaines traductions qui ne sont pas toujours très rigoureuses. Deux exemples : Dans les passages platoniciens qu'elle cite p. 36-37 (Politique, 272c-d; 274c-d; Lois, 677a-679c), on cherche vainement - sauf erreur de ma part - «l'expression thèriôdès bios », que Platon aurait récupérée «des sophistes "évolutionnistes" pour mieux s'opposer à leur vision de l'histoire ", une expression qui, "dans la conception » du philosophe, "n'a rien de proprement bestial».

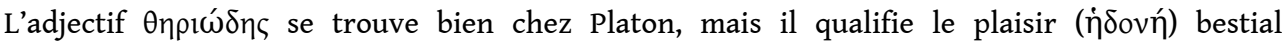
(République, IX, 591c) ; ou encore, Platon emploie, dans le Cratyle (394e), l'expression tò $\theta \eta p i \tilde{\omega} \delta \varepsilon \varsigma$

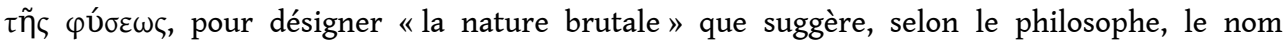
d'Oreste. D'autre part, Dionysos Anthroporrhaistês n'est pas, sur l'île de Tenedos, un dieu Démembreur d'humains, comme le dit A. Nagy (p. 92 avec la note 48), en suivant tout simplement cette fausse traduction répétée par les modernes, en alternance avec le Dépeceur d'hommes. L'analyse lexicale désigne ce Dionysos plutôt comme un Marteleur d'humains, comme celui qui «brise » les hommes avec un marteau (rhaistêr), il ne les "démembre" pas (diamelizô). Qui plus est, 
le veau nouveau-né que les gens de Tenedos offrent à ce dieu n'est pas «mis en pièces ", comme l'écrit l'auteure en renvoyant à Élien. Tout d'abord, il ne s'agit pas des Histoires variées (III, 42) d'élien, citées sans doute par inadvertance, mais de la Nature des animaux (XII, 34), où Élien mentionne justement le culte de Dionysos Anthroporrhaistês. Or ce passage dit clairement que les Tenedioi « sacrifient » (katathuousin) le petit veau, il n'y est pas question de "démembrement" ou de "dépeçage" de l'animal (je me permets de renvoyer ici à un article susceptible d'apporter quelques précisions sur ce sacrifice particulier : «Sacrificing to Dionysos : Regular and Particular Rituals », in R. SCHLESIER [éd.], A Different God ? Dionysos and Ancien Polytheism, Berlin/Boston, 2011, p. 47-60).

6. J'avais essayé, il y a longtemps, de discuter certaines thèses de Pierre Bonnechere, en particulier la question du sacrifice humain comme " une valeur d'inversion », le problème de la mort sacrificielle de jeunes gens en tant que mort «initiatique ", l'immolation des humains comme le propre des barbares etc. («À propos du sacrifice humain en Grèce ancienne: remarques critiques », dans Archiv für Religionsgeschichte 1 [1999], p. 61-82). Entretemps, d'autres écrits de l'auteur viennent nourrir le thème du sacrifice humain (voir la Bibliographie sélective dans le présent volume), ce qui montre que notre dialogue mutuel et toujours amical a encore de l'avenir.

7. Artémis n'est pas d'ailleurs la seule divinité grecque qui exige ou accepte volontiers l'immolation d'un être humain sur ses autels, ce qui contredit l'idée, énoncée par certains, que le sacrifice humain est décidément " non grec ».

8. Voir R. Goossens, Euripide et Athènes, Bruxelles, 1962, p. 581.

9. Dans une excellente étude, non citée par J. Bremmer : «Grecs et Barbares dans les tragédies d'Euripide. La fin des différences? ", Ktema 9 (1984), p. 27-53.

10. Je pense toujours que presbutatos désigne ici « le plus âgé » des descendants d'Athamas, «the eldest of that family ", comme il a été justement traduit dans ce passage d'Hérodote (p. 102), et non pas «the eldest son of the group ", comme l'écrit, ensuite, l'A. à la p. 111, ce qui n'est pas la même chose (c'est moi qui souligne ; cf., sur cette question, l'étude citée à la note $6, \mathrm{p} .73$ ).

11. Il s'agit de la traduction française légèrement augmentée d'un texte paru en allemand en 2006, in B. SEIDENSTICKER, M. VOHLER (éd.), Gewalt und Ästhetik. Zur Gewalt und ihrer Darstellung in der griechischen Klassik, Berlin/New York, 2006, p. 59-87.

12. Comme, par exemple, la théorie de la "culpabilité", qu'A. Henrichs met justement en doute. La fameuse inscription de Sélinonte et le sarcophage de Polyxène (examiné en détail, comme on a $\mathrm{vu}$, par I. Mylonopoulos) lui offrent aussi l'occasion de mettre en question la distinction moderne entre "olympiens" et "chthoniens" (p. 125, 129).

13. Pausanias IX, 8, 1-2 (et non pas $X, 8,1-2$, comme il a été écrit par inadvertance).

14. Ce que suggère le poème même, en les qualifiant de «meilleurs ».

15. D'ailleurs, à quelle puissance ce sacrifice serait-il adressé ?

16. Ces deux constatations générales doivent être beaucoup nuancées, car une série de cas grecs, par exemple, ne renvoient ni à « l'Autre ", ni au " passé ».

17. C'est moi qui souligne. Sur ce point important, voir ci-dessus, la contribution de Robert Parker qui exprime une opinion différente.

18. Cette thèse fait partie de ce qu'on peut appeler, en général, la "quête des origines ", une quête souvent aléatoire, mais qui satisfait sans doute le besoin de remplir les "cases" de notre histoire humaine. Soyons cependant modestes, et admettons qu'il s'agit d'une hypothèse qu'on peut toujours soumettre au débat.

19. Voir son ouvrage de référence, L'ordalie dans la Grèce primitive, Paris, 1904.

20. Cf. des cas analogues, en Grèce ancienne, lorsqu'on exige parfois de choisir une victime humaine de " bonne naissance ", appartenant à une famille noble, illustre.

21. Cela fait penser à ces victimes grecques, mais animales en l'occurrence, qu'on garde et nourrit pendant un certain temps, avant de les sacrifier sur les autels divins. 
22. Ce qui ne manquera pas de rappeler aux hellénistes le cas des apheta ou aneta zôia, des animaux qui errent libres, sans liens, sans attaches, et qui servent parfois de victimes sacrificielles - mais dans un contexte différent de celui de l'Inde.

23. Plus de 2000 inhumations, dont on a distingué seize tombes qualifiées de "royales ", qui remonteraient aux $\mathrm{XXVII}^{\mathrm{e}}-\mathrm{XXIII}{ }^{\mathrm{e}}$ siècles avant notre ère.

24. Les morts d'accompagnement, La servitude volontaire, I, Paris, 2004.

\section{AUTEUR}

\section{STELLA GEORGOUDI}

EPHE (Paris) - Unité de recherche AnHiMA

stgeorg@ehess.fr 